\title{
Los determinantes de la compra de productos con etiquetas de contenido social y ambiental
}

\author{
Isabel Carrero, Carmen Valor y Raquel Redondo
}

\section{RESUMEN}

Las empresas social y medioambientalmente responsables realizan grandes inversiones para obtener los sellos que las certifiquen como tales. De cara a diferenciarse en el mercado, los logos o etiquetas responsables son considerados como un vehículo eficaz para comunicar al consumidor los atributos sociales y ambientales de los productos. Sin embargo, estudios previos concluyen que la mayoría de los consumidores no las conocen, no las entienden o no creen en ellas. Mientras que trabajos anteriores han examinado la influencia de las actitudes en la compra de productos con etiquetas, este trabajo analiza la influencia de la comprensión y de variables asociadas a la motivación en la compra de productos con etiquetado responsable. A partir de entrevistas estructuradas a 289 consumidores en Madrid, se concluye que el conocimiento de la etiqueta es un antecedente clave de la compra de productos con atributos éticos. Así, las organizaciones interesadas en el marcado RSE deben invertir en comunicación orientada a construir el conocimiento de la etiqueta. A partir de los resultados encontrados, se formulan sugerencias para profesionales y académicos interesados en el marketing de productos con etiquetado a contenido social y ambiental.

PALABRAS CLAVE: Responsabilidad social corporativa (RSC), marcado o etiquetado socialmente responsable, conocimiento de la etiqueta, sostenibilidad, España.

CLAVES ECONLIT: D830, M140, M310.

Cómo citar este artículo: CARRERO, I., VALOR, C. \& REDONDO, R. (2015): "Los determinantes de la compra de productos con etiquetas de contenido social y ambiental", CIRIEC-España, Revista de Economía Pública, Social y Cooperativa, 83, 235-250.

Correspondencia: Universidad Pontificia Comillas-ICADE. C/. Alberto Aguilera, 23. 28015 Madrid, España, Tel: 9154228 00. E-mails de contacto: icarrero@cee.upcomillas.es, cvalor@cee.upcomilla.es, rredondo@cee.upcomillas.es. 


\title{
Les facteurs déterminants de l'achat de produits à étiquettes à contenu social et environnemental
}

RÉSUMÉ : Les entreprises à caractère social et environnemental investissent considérablement pour obtenir les labels les certifiant comme telles. Pour se distinguer sur le marché, les logos ou étiquettes responsables apparaissent comme un moyen efficace de communiquer au consommateur les attributs à caractère social ou environnemental des produits. Cependant, des études antérieures concluent que la plupart des consommateurs ne les connaissent pas, ne les comprennent pas ou n'y croient pas. Des travaux précédents ayant examiné l'influence des comportements sur l'achat de produits étiquetés, cette étude analyse l'influence de la compréhension et de variables associées à la motivation dans l'achat de produits à étiquetage responsable. Sur base d'interviews structurées auprès de 289 consommateurs à Madrid, on conclut que la connaissance de l'étiquette est un facteur clé dans l'achat de produits éthiques. Les sociétés intéressées par la certification RSE doivent donc investir dans une communication axée sur la construction de la connaissance de l'étiquette. Les résultats de l'étude permettent de formuler des propositions à l'attention des professionnels et des chercheurs intéressés par le marketing de produits dont l'étiquetage est à contenu social et environnemental.

MOTS CLÉ : Responsabilité sociale des entreprises (RSE), marquage ou étiquetage socialement responsable, connaissance de l'étiquette, durabilité, Espagne.

\section{Decisive factors for purchasing products with social and environmental labels}

\begin{abstract}
Socially and environmentally responsible firms make great efforts to obtain the labels that certify their social and environmental standards. To differentiate them from their competitors, the so-called 'socially responsible' labels are considered the best way to inform consumers of their social and environmental performance. However, previous studies have concluded that most consumers do not recognize, do not understand or do not trust labels. Whereas previous studies have examined the influence of attitudes on the purchase of labelled products, this study analyses the influence of understanding and of variables associated with motivation to purchase such products. On interviewing 289 consumers in Madrid, it was found that knowledge of the label is a key precursor to CSR purchasing. The paper concludes by suggesting that organizations and marketing professionals with an interest in the CSR-labelled market should invest in building up familiarity with the label. Avenues for academic research are also proposed.
\end{abstract}

KEY WORDS: Corporate social responsibility (CSR), labelling and branding, label knowledge, social and environmental labels, sustainability, Spain. 


\section{1.- Introducción1}

Cada vez es mayor el número de empresas que deciden contribuir al desarrollo económico tomando en cuenta los intereses de sus stakeholders y asegurando que su actividad no causa ningún perjuicio social o medioambiental. En esta línea, la Unión Europea promueve la Responsabilidad Social de la Empresa (en adelante RSE) como una manera de alcanzar el triple objetivo estratégico del crecimiento económico, competitividad y cohesión social (Comisión Europea, 2006).

Las empresas que deciden ser socialmente responsables deben hacer una apuesta firme para conseguirlo, asegurando que su actividad se lleve a cabo respetando estos criterios de sostenibilidad. Someterse a procesos de certificación externa para avalarlo resulta costoso, no sólo en términos económicos sino también de dedicación de tiempo y recursos humanos. Siendo así, es lógico que las marcas responsables busquen un reconocimiento por parte del mercado.

La RSE es considerada una fuente de ventajas competitivas para la empresa ya que se asume que hay incentivos económicos para su desarrollo: siendo responsable, la empresa atrae y retiene clientes, lo que se traduce en un incremento de ventas que llevará, a su vez, a un aumento de beneficios (Webb, Mohr y Harris, 2008). Esta idea parte de la premisa de que los consumidores comprarán de forma responsable, esto es, que tendrán en cuenta el desempeño social y medioambiental de las marcas al tomar su decisión de compra (Comisión Europea, 2006; Parlamento Europeo, 2006).

Sin embargo, incluso si los consumidores quieren tener en cuenta estos atributos, no siempre pueden hacerlo, porque no tienen información para realizar esta elección (Fliess, Lee, Dubreuil y Agatiello, 2007; Uusitalo y Oksanen, 2004; Valor, 2007). Por eso, dar esta información al mercado es clave para poder construir ventajas competitivas en torno a la RSE.

Las etiquetas sociales y ambientales, también llamadas etiquetas de RSE, se consideran la mejor manera de indicar que un producto o marca se alinea a las expectativas sociales y ambientales de los consumidores (Chakrabarty y Grote 2009; De Pelsmacker Janssens, Sterckx y Mielants, 2005; Drichoutis, Lazaridis y Nayga, 2006; Fliess et al., 2007; Uusitalo y Oksanen, 2004). También la Comisión Europea y los gobiernos nacionales (Comisión Europea, 2006) las consideran la mejor manera de empoderar a los consumidores responsables porque suponen un medio de diferenciación en el mercado que favorece a las empresas responsables (McEachern y Warnaby, 2008) y por esta razón están promoviendo su uso. Por citar algunos ejemplos, son etiquetas RSE, en el caso de alimentación, las

1.- Agradecemos el apoyo financiero del Ministerio de Trabajo e Inmigración (BOE 17-02-2010) para realizar esta investigación, así como a José Manuel Rosa por su ayuda en la recolección de información. 
etiquetas ecológicas, tanto nacionales como autonómicas, o la de comercio justo. En caso de productos de droguería, los certificados Ecocert o Leaping Bunny.

Sin embargo, estudios previos demuestran que estas etiquetas están fallando en su objetivo de dotar al consumidor de información en el punto de venta; así, no están siendo una herramienta de diferenciación para las marcas responsables: los consumidores no las reconocen, no las entienden, 0 no creen que sean ciertas las afirmaciones que leen (Carrero y Valor, 2012; Flies et al., 2007). Esto puede explicar las bajas cuota de mercado de las marcas con etiquetado RSE y las diferencias tan grandes encontradas entre los consumidores que manifiestan el deseo de comprar responsable y los que efectivamente lo hacen (De Pelsmacker y Janssens, 2007; De Pelsmacker et al., 2005; François-Lecompte y Valette-Florence, 2006; Shaw, Hogg, Wilson, Shiu, y Hassan, 2006; Uusitalo y Oksanen, 2004).

Ningún estudio ha analizado los determinantes de la compra de productos con etiquetado de contenido social y medioambiental en España. Los estudios sobre etiquetas de RSE han analizado la influencia de la actitud hacia el etiquetado en la compra (De Pelsmacker y Janssens, 2007; McEachern y Warnaby, 2008) y/o han tratado conocer el perfil sociodemográfico de sus usuarios (D'Souza, Taghian, Lamb y Peretiatko, 2007) sin llegar a conclusiones definitivas sobre qué criterios sociodemográficos están más asociados al consumidor de etiquetas de RSE. Además, estos trabajos se han centrado en dos etiquetas: comercio justo y productos orgánicos.

Este trabajo adopta una perspectiva amplia en la consideración de etiquetas éticas, analizando 11 etiquetas de RSE. Además, guiados por el modelo MOA (motivación, oportunidad y capacidad, por sus siglas en inglés), se plantea que los antecedentes clave de la compra de productos con etiqueta serán variables asociadas a la motivación (motivación por estar informado, motivación para usar atributos éticos en la compra e importancia dada al precio) y la capacidad del individuo, entendida como comprensión de la etiqueta.

Para conseguir este objetivo, se realizó una encuesta personal a 385 consumidores responsables de compra del hogar, en la que se preguntaba sobre el conocimiento y uso de las etiquetas de RSE más frecuentes en los productos de alimentación y droguería en España (Valor y Calvo, 2009). De esas encuestas se obtuvieron un total de 289 registros completos. 


\section{2.- Los determinantes de la compra de productos con etiquetas de contenido social y medioambiental (etiquetas RSE)}

Stigler (1961), en su modelo económico de la búsqueda de información, argumenta que la información sobre un producto es un bien económico cuya producción y obtención tiene un coste de tiempo y esfuerzo para el consumidor. Este modelo asume que los consumidores realizan un proceso activo de búsqueda de información que concluirá cuando el coste y beneficio de esta información estén en equilibrio.

Algo parecido ocurre en el caso de productos con etiquetado RSE: para conocer este etiquetado el consumidor debe hacer necesariamente una búsqueda de información previa. Por eso, en este artículo se plantea que el conocimiento, más que las actitudes, son un factor determinante de la compra de productos con etiquetado de RSE.

El consumidor debe hacer una búsqueda previa a la compra por dos razones. En primer lugar, porque este tipo de etiquetas no ofrece información completa a los consumidores; por el contrario, para entender las etiquetas, es necesario algo de conocimiento previo (Thøgersen, Haugaard y Olesen, 2010). Por ejemplo, si un consumidor lee: Marine Stewardship Council2 y ve el logotipo correspondiente, es poco probable que pueda inferir con precisión el atributo protegido y el emisor de la etiqueta. En segundo lugar, es difícil que este conocimiento se construya en la tienda, si se tiene en cuenta el elevado número de etiquetas -se estima que existen más de 200 sólo en Europa (De Pelsmacker et al. 2005) - y que su diseño tiende a confundir a los consumidores, en lugar de ayudar a entender (van Amstel, Driessen y Glasbergen, 2008). Hay más de una veintena de sistemas nacionales de etiquetado ecológico, además de cientos de otros con un enfoque específico como la pesca sostenible o la producción orgánica. Más aún, las etiquetas son visualmente parecidas, aunque los requisitos para su obtención puede variar mucho (Fliess et al. 2007). Es difícil saber qué tipo de requisitos debe cumplir la empresa, quién otorga la etiqueta, y cuáles son las diferencias con otros programas similares a menos que la búsqueda se lleve a cabo antes de comprar.

Varios autores han determinado que la conducta humana se explica por tres grandes factores: motivación, oportunidad y capacidad (llamado MOA theory por sus siglas en inglés motivation-opportunity-ability). Este marco ha sido aplicado para explicar la influencia de la publicidad (Batra y Ray, 1986; Maclnnis, Moorman y Jaworski, 1991) y otras formas de comunicación (Hallahan, 2001), así como para explicar el consumo sostenible (Olander y Thogersen, 1995). 
En este trabajo entendemos que las etiquetas dan la oportunidad al consumidor de comprar de forma responsable. En el marco MOA, se define oportunidad como la atención fijada en el objeto; si hay distracciones, se entiende que no ha habido oportunidad (Maclnnis et al., 1991). Esta variable así definida no es analizada en este trabajo, sino que se sigue a otros autores que combinan oportunidad y habilidad, bajo la misma rúbrica de capacidad (Andrews, 1988). En otras palabras, siguiendo el modelo MOA se plantea que para que haya compra efectiva es necesario que el consumidor esté motivado y que sea capaz de entender la información que proyecta la etiqueta.

Así, la comprensión o conocimiento de la etiqueta se plantea como una medida de capacidad: si comprende la etiqueta puede descifrar la información que contiene (Maclnnis et al., 1991).

$H_{1}$. Existe una relación positiva entre el conocimiento de la etiqueta y la compra de productos etiquetados RSE.

Además, hay una relación entre motivación y capacidad. Las teorías sobre procesamiento de la información (Petty et al., 1981; Petty y Cacioppo, 1984; Zuckerman y Chaiken, 1998) han encontrado evidencia de que a mayor motivación, mayor búsqueda de información lo que llevará a mayor capacidad, tal como se define en este trabajo. Igualmente, estudios sobre etiquetado RSE han encontrado que solo los consumidores motivados hacen un esfuerzo por construir el conocimiento necesario para utilizar las etiquetas de RSE (Thøgersen et al., 2010).

Según la teoría de elaboración de la información (Petty et al., 1981; Petty y Cacioppo, 1984) hay que diferenciar dos elementos en la motivación: la motivación por estar informado (nivel general) y el interés personal en un tema (nivel específico, que otros autores llaman implicación) (Andrews, 1988). En este caso, la motivación por estar informado se define como el uso del etiquetado como fuente de información (Drichoutris et al., 2006) y el interés por el tema se corresponde con el hecho dar mayor peso a los atributos éticos a la hora de tomar la decisión de compra. Si un consumidor está interesado en los atributos éticos, buscará mayor información comparativamente a los consumidores no interesados 0 apáticos. Así, se propone que:

$\mathrm{H}_{2}$ a. Hay una relación positiva y significativa entre la importancia dada al etiquetado general y el conocimiento de la etiqueta.

$\mathrm{H}_{2}$ b. Hay una relación positiva y significativa entre la importancia dada a los atributos RSE y el conocimiento de la etiqueta.

Por otro lado, los consumidores más sensibles al precio tienden a estar menos motivados a recabar información sobre las etiquetas de los productos, puesto que para ellos el precio es el elemento clave en el que fundamentar su compra (Drichoutis et al., 2006; Nayga, Lipinski y Savur, 1998). De hecho, estudios como el de D'Souza et al. (2006) encontraron que los consumidores más preocupados por el precio son los que menos leen las etiquetas ambientales. 
$\mathrm{H}_{3}$. Hay una relación negativa y significativa entre la importancia dada al precio y el conocimiento de la etiqueta.

La figura 1 refleja el modelo conceptual que recoge las hipótesis planteadas.

\section{Figura 1. Modelo conceptual completo de los factores determinantes de la compra de productos con marca o etiqueta de contenido social y medioambiental (etiquetas RSE)}

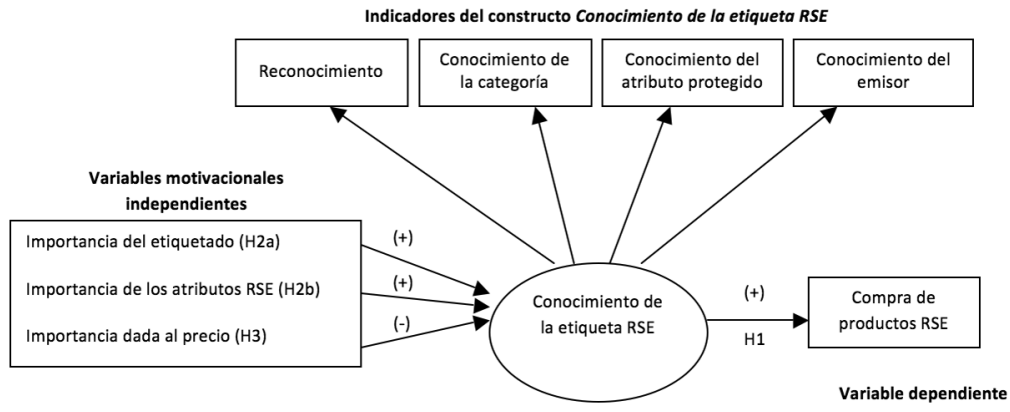

\section{3.- Metodología}

Para testar el modelo se realizaron entrevistas personales, mediante cuestionario estructurado con preguntas cerradas, a los consumidores encargados de realizar la compra del hogar mayores de edad, siguiendo muestro estratificado por sexo, edad y renta, este último mediante la localización de informantes en centros comerciales situados en distritos con diferente nivel de renta. Los entrevistados fueron elegidos aleatoriamente a la salida de centros comerciales de distintas zonas del área metropolitana de Madrid y el trabajo de campo tuvo lugar en noviembre del 2010. Se entrevistaron un total de 385 individuos (error muestral del 5,5\% para $p=q=50 \%$ ), pero la muestra válida para el análisis de 289 casos. La muestra es representativa de la Comunidad de Madrid en cuanto a renta, edad y sexo del responsable de compras, como se muestra en la tabla 1. 


\section{Tabla 1. Datos socio-demográficos de la muestra, cifras en porcentajes}

\begin{tabular}{|c|c|c|c|c|}
\hline Sexo & \multicolumn{2}{|c|}{$\begin{array}{c}\text { Hombre } \\
34\end{array}$} & \multicolumn{2}{|c|}{$\begin{array}{c}\text { Mujer } \\
66\end{array}$} \\
\hline Edad & $\begin{array}{c}18-29 \text { años } \\
20,5\end{array}$ & $\begin{array}{c}\text { 30-49 años } \\
52,8\end{array}$ & $\begin{array}{c}\text { 50-65 años } \\
21,2\end{array}$ & $\begin{array}{c}\text { Más de } 65 \text { años } \\
5,4\end{array}$ \\
\hline Nivel educativo & $\begin{array}{c}\text { Sin estudios } \\
6,5\end{array}$ & $\begin{array}{c}\text { Primarios } \\
11,5\end{array}$ & $\begin{array}{c}\text { Secundarios } \\
41,1\end{array}$ & $\begin{array}{c}\text { Universitarios } \\
40,3\end{array}$ \\
\hline
\end{tabular}

El cuestionario fue elaborado a partir de variables e indicadores fruto de una revisión de estudios anteriores.

El Conocimiento de la etiqueta es medido a partir de diferentes variables: reconocimiento, conocimiento de atributo protegido, de la categoría y del emisor. En primer lugar, el reconocimiento: si los consumidores no son capaces de reconocer una etiqueta, es muy probable que no entiendan su significado. Así, la notoriedad o reconocimiento de la etiqueta es la primera variable del factor conocimiento. Por otra parte, conocer su significado implica conocer el tema 0 atributo protegido por la etiqueta. Finalmente, conocer la categoría de producto donde se puede encontrar y el conocimiento del emisor (Bonroy y Lemarie, 2008; De Pelsmacker et al., 2005; D'Souza et al., 2007) es otro indicador del conocimiento de la misma.

La tabla 2 desglosa las variables empleadas en el modelo, los indicadores de medición y las fuentes en las que se apoyan. 


\section{Tabla 2. Variables empleadas en el modelo e indicadores de medición}

\begin{tabular}{|c|c|}
\hline Medición de la variable & Medición de la variable \\
\hline VARIABLES DEL CONSTRUCTO CONOCIMIENTO DE LA ETIQUETA & \\
\hline $\begin{array}{l}\text { Reconocimiento: notoriedad, número de etiquetas reconocidas (valor de } 0 \text { a 12) } \\
\text { (Orgánico europeo, Orgánico español, Orgánico andaluz, WWF-ADENA, Comercio } \\
\text { Justo, Ecolabel, Sustainable Cleaning, Rainforest Alliance, FSC, Marine Stewardship } \\
\text { Council, Leaping Bunny y Ecocert). }\end{array}$ & $\begin{array}{l}\text { Selección de etiquetas a partir del inven- } \\
\text { tario realizado por Valor y Calvo (2009) } \\
\text { en distribuidores del municipio de } \\
\text { Madrid. }\end{array}$ \\
\hline $\begin{array}{l}\text { Conocimiento de la categoría: suma de las etiquetas de las que conoce la cate- } \\
\text { goría en la que está presente dividido por el número de etiquetas que reconoce (varia- } \\
\text { ble continua con valores de } 0 \text { a } 1 \text { ). } \\
\text { Conocimiento del atributo protegido: suma de las etiquetas de las que conoce el } \\
\text { atributo al que se refiere la etiqueta dividido por el número de etiquetas que reconoce } \\
\text { (variable continua con valores de } 0 \text { a } 1 \text { ). } \\
\text { Conocimiento del emisor: suma de las etiquetas de las que conoce quien otorga } \\
\text { la etiqueta dividido por el número de etiquetas que reconoce (variable continua con } \\
\text { valores de } 0 \text { a 1). }\end{array}$ & $\begin{array}{l}\text { Bonroy y Lemarie, 2008; De Pelsmacker } \\
\text { et al., 2005; D'Souza et al., 2007; } \\
\text { McEachern y Warnaby, } 2008\end{array}$ \\
\hline ACTO DE COMPRA EFECTIVO & \\
\hline $\begin{array}{l}\text { Compra: suma de productos con etiquetas RSE que ha comprado en el último mes } \\
\text { (valor de } 0 \text { a 12) }\end{array}$ & Baltas, 2001 \\
\hline VARIABLES RELACIONADAS CON MOTIVACIÓN & \\
\hline $\begin{array}{l}\text { Importancia dada al etiquetado: valor de la respuesta a la pregunta "En su opinión } \\
\text { ¿cómo de importante es la información que aparece en el envase del producto a la } \\
\text { hora de tomar su decisión de compra?" Pregunta de escala Likert de } 11 \text { posiciones. } \\
\text { Importancia dada a atributos de RSE: suma de los puntos otorgados a los atribu- } \\
\text { tos éticos (impacto ambiental, bienestar animal y condiciones sociales) en estas dos } \\
\text { preguntas (valor de } 0 \text { a 18). } \\
\text { "De estos atributos, ¿podría ordenar los } 3 \text { más importantes para usted a la hora de } \\
\text { tomar su decisión de compra de alimentos y bebidas siendo } 3 \text { el de mayor impor- } \\
\text { tancia? Marca - precio - sabor - propiedades nutricionales - impacto ambiental - } \\
\text { bienestar animal - condiciones sociales" } \\
\text { "De estos atributos, ¿podría ordenar los } 3 \text { más importantes para usted a la hora de } \\
\text { tomar su decisión de compra de droguería siendo } 3 \text { el de mayor importancia? Marca } \\
\text { - precio - ingredientes - impacto ambiental - bienestar animal - condiciones socia- } \\
\text { les". }\end{array}$ & $\begin{array}{l}\text { Baltas, 2001; De Pelsmacker y } \\
\text { Janssens, 2007; Drichoutris et al., 2006; } \\
\text { D’ Souza et al., 2001; Nayga et al., 1998; } \\
\text { Thøgersen et al., } 2010\end{array}$ \\
\hline
\end{tabular}


El análisis se realizó a través de un modelo de ecuaciones estructurales (SEM). De acuerdo con Anderson y Gerbing (1988), en primer lugar se ha testado el modelo de medida, a través de un análisis factorial confirmatorio para asegurar la unidimensionalidad, fiabilidad y validez de las escalas propuestas para el constructo conocimiento. Después, se ha procedido a testar el modelo estructural explicativo de la compra de productos con etiquetado RSE, para poder analizar el cumplimiento de las hipótesis. Con objeto de modelizar la correlación existente entre las variables explicativas de motivación (Importancia dada al etiquetado, Importancia dada a atributos de RSE e Importancia dada al precio) se han incorporado las correspondientes covarianzas en el modelo. Para todo ello, se ha utilizado el programa AMOS.

\section{4.- Resultados}

La tabla 3 precede a la presentación de los resultados del estudio. Esta tabla concentra los estadísticos descriptivos de las variables del modelo.

\section{Tabla 3. Estadísticos descriptivos de las variables del modelo}

\begin{tabular}{|l|cccc|}
\hline & Mín. & Máx. & Media & Desv. típ. \\
\hline Variables del factor conocimiento & & & & \\
Reconocimiento & 0,00 & 10,00 & 1,9965 & 1,72099 \\
Conocimiento de la categoría & 0,00 & 1,00 & 0,3986 & 0,41719 \\
Conocimiento del atributo protegido & 0,00 & 1,00 & 0,2080 & 0,32344 \\
Conocimiento del emisor & 0,00 & 1,00 & 0,0852 & 0,21118 \\
Variables asociadas a la motivación & & & & \\
Importancia dada al precio & 0,00 & 6,00 & 4,1765 & 1,78536 \\
Importancia dada a atributos de RSE & 0,00 & 12,00 & 1,0969 & 1,99938 \\
Importancia dada al etiquetado & 1,00 & 5,00 & 2,9446 & 1,10100 \\
Otras variables & & & & \\
Compra (acto consumido de) & 0,00 & 7,00 & 0,5121 & 0,97931 \\
\hline
\end{tabular}

Para el factor Conocimiento, los resultados del análisis factorial confirmatorio se muestran en la tabla 4 y son satisfactorios. La unidimensionalidad del constructo queda garantizada a través de las buenas medidas de ajuste en el modelo de medida: a pesar de que el valor de la Chi cuadrado con 2 
grados de libertad muestra un p-valor de 0,001, dado que los valores de este estadístico se ven afectados notablemente por pequeño tamaño de la muestra, se han calculado otros índices de ajuste global que refrendan la citada unidimensionalidad (GFI=0,978; $\mathrm{AGFI}=0,891 ; \mathrm{NFI}=0,961 ; \mathrm{IFI}=0,966$; $\mathrm{CFI}=0,966 ; \mathrm{SRMR}=0,034)$. Por su parte, la fiabilidad compuesta del constructo toma un valor de 0,8 , que constata la fiabilidad del mismo. La validez convergente se avala al ser todos los indicadores significativos y con altas cargas factoriales, así como la validez discriminante, que se examina con la Average Variance Extracted (AVE) (Babin, Darden y Griffin, 1994), que alcanza un valor de 0,5. De acuerdo con Dillon y Goldstein (1984) un valor de 0,5 o superior indica que la validez del constructo y las variables individuales es alta.

\section{Tabla 4. Resultados del análisis factorial confirmatorio}

\begin{tabular}{|l|c|cc|}
\hline & Cargas factoriales & \multicolumn{2}{|c|}{ Índices ajuste } \\
\hline Reconocimiento & 0,532 & GFI & 0,978 \\
Conocimiento de la categoría & 0,743 & AGFI & 0,891 \\
Conocimiento del atributo protegido & 0,867 & NFI & 0,961 \\
Conocimiento del emisor & 0,634 & IFI & 0,966 \\
AVE & 0,5 & CFI & 0,966 \\
Fiabilidad conjunta & 0,8 & SRMR & 0,034 \\
\hline
\end{tabular}

El modelo estructural se ha testado para analizar las hipótesis H1 hasta H3. Los resultados del modelo aparecen en la figura 2 (sobre las hipótesis aparecen los efectos directos estandarizados y en las variables endógenas del modelo, entre paréntesis, su varianza explicada). El ajuste global del modelo es bueno ( $\mathrm{GFI}=0,965 ; \mathrm{AGFI}=0,903 ; \mathrm{NFI}=0,945 ; \mathrm{IFI}=0,960 ; \mathrm{CFI}=0,960 ; \mathrm{SRMR}=0,041$ ) y todos los coeficientes estimados en el mismo son significativos y con el signo esperado, por lo que se verifica el cumplimiento de las hipótesis propuestas. Así, se constata el hecho de que el conocimiento de las etiquetas RSE incide positiva e intensamente en la compra de esos productos (H1). Además, tanto la importancia dada al etiquetado como a los atributos RSE manifiestan una relación causal y positiva sobre el conocimiento de dichas etiquetas ( $\mathrm{H} 2 \mathrm{a}, \mathrm{H} 2 \mathrm{~b})$. Finalmente, la importancia que los consumidores dan al precio representa un freno al conocimiento de las etiquetas (H3). De esta manera, nuestro estudio encuentra que las variables asociadas a la motivación inciden significativamente en el conocimiento de las etiquetas RSE y éste, de manera especialmente intensa, es un factor determinante en la compra de las mismas. 


\section{Figura 2. Estimación del modelo estructural (coeficientes estandarizados)}

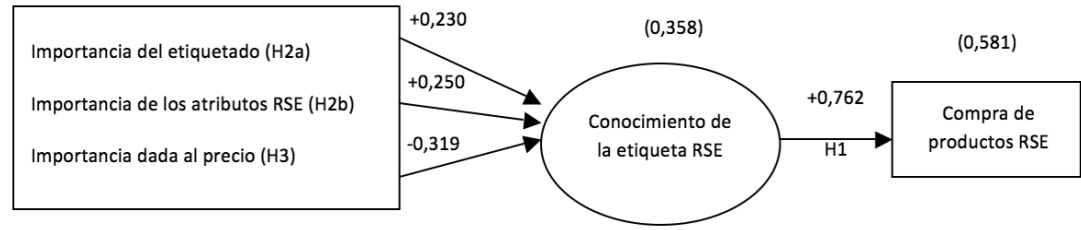

\section{5.- Conclusiones}

El etiquetado RSE debe cumplir una doble función: desde el lado de la demanda se presenta como una forma eficaz de reducir las asimetrías de información y dar poder al consumidor y, desde la oferta, es una forma de reconocimiento y ventaja competitiva para aquellas empresas comprometidas social y medioambientalmente. Sin embargo, los datos y estudios previos parecen indicar que estas etiquetas están fallando en su cometido. Este trabajo tenía como objetivo testar si el conocimiento y variables asociadas a la motivación eran antecedentes de la compra de etiquetas RSE, siguiendo los modelos teóricos del modelo económico de búsqueda de información y el modelo MOA.

Efectivamente, los resultados sugieren que variables relacionadas con la motivación, como son el interés por la información del etiquetado, la importancia dada a los atributos RSE y la menor importancia dada al precio, influyen en el conocimiento sobre el etiquetado de RSE y éste en el acto de compra. Así, los consumidores más motivados son los que adquieren un mayor conocimiento sobre las etiquetas y, los que más conocen, los que más las compran.

Se concluye pues que la compra depende en buena media del conocimiento. En consecuencia, obtenida la etiqueta, la marca debe informar y formar al consumidor. La notoriedad o reconocimiento de la etiqueta es una medida parcial del conocimiento, pero por sí misma es insuficiente: el simple reconocimiento de la etiqueta no basta. Es preciso que el consumidor reconozca la etiqueta y también el ramillete de atributos que ésta protege, en qué categorías de producto se puede encontrar y quién la otorga. Por tanto, las organizaciones que potencian sus atributos RSE en el mercado de gran consumo deben invertir en comunicación orientada a construir conocimiento sobre las etiquetas. Quizá así se reduzca la brecha existente entre actitud favorable a los sellos y la compra del producto (De 
Pelsmacker y Janssens, 2007; De Pelsmacker et al., 2005; François-Lecompte y Valette-Florence, 2006; Shaw, Hogg, Wilson, Shiu, y Hassan, 2006; Uusitalo y Oksanen, 2004). A pesar de que los consumidores tienen una voluntad de consumir responsablemente, sólo aquellos que tienen un alto grado de conocimiento de los atributos que protege el sello o de los sellos en sí mismos son capaces de reconocerlos y de comprarlos.

Aunque la formación del consumidor es una tarea encomendada a los poderes públicos, lo cierto es que, en la situación actual, es recomendable que las empresas con atributos sociales y ambientales dediquen su comunicación a esta tarea. Construir conocimiento puede hacerse de varias maneras: individualmente, aliándose con distribuidores, especialmente con aquellos que incorporan más productos certificados en sus lineales, para dar información en el punto de venta. Colectivamente, colaborando con organizaciones sociales (por ejemplo, con las que promueven el Comercio Justo como forma de cooperación con el sur productor) para hacer llegar a asociados esta información. Ante la falta de inversión pública en esta materia, las alianzas parecen una forma eficaz de conseguir el objetivo, sin que ninguna parte asuma la totalidad de los costes.

Con respecto a las futuras líneas de investigación que abre este trabajo, en primer lugar, este modelo podría completarse con variables de otras teorías de consumo responsable que permitieran explicar mejor el fenómeno aquí expuesto. En particular, podría introducirse una variable que se ha mencionado en literatura previa como una condición necesaria para el uso del etiquetado en general: la credibilidad (De Pelsmacker et al, 2005; Stø and Strandbakken, 2005). De hecho, y siguiendo el modelo aquí planteado, la credibilidad depende del conocimiento del emisor de la etiqueta. Es fundamental examinar cómo construyen los consumidores la credibilidad, en qué basan su juicio y cómo este juicio puede ser modificado. Por otro lado, se podrían aplicar las teorías de procesamiento de información para ver cómo se construye el conocimiento sobre el etiquetado.

Además, para profundizar en los antecedentes del constructo conocimiento podrían llevarse a cabo experimentos en laboratorio. Por último, de cara también a mejorar el modelo, este debería ser replicado para cada una de las etiquetas existentes. Para ello, debería usarse una muestra de conveniencia introduciendo un número mayor de consumidores conocedores de las etiquetas y así poder evitar varianzas tan cercanas a cero. 


\section{Referencias}

ANDERSON, J.C. \& GERBING, D.W. (1988): "Structural equation modeling in practice: A review and recommended two-step approach", Psychological Bulletin, 103(3), 411-423.

ANDREWS, J.C. (1988): "Motivation, Ability, and Opportunity to Process Information: Conceptual and Experimental Manipulation Issues", Advances in consumer research, 15(1), 219-225.

BABIN, B.J., DARDEN, W.R. \& GRIFFIN, M. (1994): "Work and/or fun: measuring hedonic and utilitarian shopping value", Journal of Consumer Research, 20, 644-656

BALTAS, G. (2001): "Nutrition labelling: issues and policies", European Journal of Marketing, 35, 708721.

BATRA, R. \& RAY, M.L. (1986): "Situational effects of advertising repetition: The moderating influence of motivation, ability, and opportunity to respond", Journal of Consumer research, 432-445.

BONROY, O. \& LEMARIE, S. (2008): "Downstream Labeling and Upstream Competition", Working Paper GAEL; 2008-06, Institut National de la Recherche Agronomique - Université Pierre Mendès France.

CARRERO BOSCH, I. \& VALOR MARTíNEZ, C. (2012): "La Relación del Consumidor con el Etiquetado Responsable", Revista de Responsabilidad Social de la Empresa, 10, Enero-Febrero, 79-104.

CHAKRABARTY, S. \& GROTE, U. (2009): "Child Labor in Carpet Weaving: Impact of Social Labelling in India and Nepal", World Development, 37(10), 1683-1693.

COMISIÓN EUROPEA (2006): Implementing the Partnership for Growth and Jobs: Making Europe a Pole of Excellence on CSR (COM(2006)136 final 22.03.2006), http://ec.europa.eu/employment_social/soc-dial/csr/index.htm.

DILLON, W.R. \& GOLDSTEIN, M. (1984): Multivariate Analysis-Methods and Applications, Wiley, New York.

D'SOUZA, C., TAGHIAN, M., LAMB, P. \& PERETIATKO, R. (2007): "Green Decisions: Demographics and Consumer Understanding of Environmental Labels", International Journal of Consumer Studies, 31(4), 371-376.

DE PELSMACKER, P., JANSSENS, W., STERCKX, E. \& MIELANTS, C. (2005): "Consumer Preferences for the Marketing of Ethically Labelled Coffee", International Marketing Review, 22(5), 512-530. 
DE PELSMACKER, P. \& JANSSENS, W. (2007): "A model for fair trade buying behaviour: The role of perceived quantity and quality of information and of product-specific attitudes", Journal of Business Ethics, 75(4), 361-380.

DRICHOUTIS, A.C., LAZARIDIS, P. \& NAYGA, R.M. (2006): "Consumers' Use of Nutritional Labels: A Review of Research Studies and Issues", Academy of Marketing Science Review, 9, en http://www.allbusiness.com/marketing-advertising/4089043-1.html.

FLIESS, B., LEE, H.Y., DUBREUIL, O.L. \& AGATIELLO, O. (2007): "CSR and Trade: Informing Consumers about Social and Environmental Conditions of Globalised Production", Trade Policy Working Paper No. 47 - PART I, Working Party of the Trade Committee TD/TC/WP(2006):17/FINAL.

FRANÇOIS-LECOMPTE, P. \& VALLETTE-FLORENCE, A. (2006): "Mieux connaître le consommateur socialement responsible", Décisions Marketing, 41, 69-79.

HALLAHAN, K. (2001): "Enhancing motivation, ability, and opportunity to process public relations messages", Public Relations Review, 26(4), 463-480.

LODZIAK, C. (2002): The Myth of Consumerism, London: Pluto Press.

MACINNIS, D.J., MOORMAN, C. \& JAWORSKI, B.J. (1991): "Enhancing and measuring consumers' motivation, opportunity, and ability to process brand information from ads", The Journal of Marketing, 32-53.

MCEACHERN, M.G. \& WARNABY, G. (2008): "Exploring the relationship between consumer knowledge and purchase behaviour of value-based labels", International Journal of Consumer Studies, 32, 414-426.

NAYGA, R.M., LIPINSKI, D. \& SAVUR, N. (1998): "Consumers' use of nutritional labels while food shopping and at home", Journal of Consumer Affairs, 32, 106-120.

ÖLANDER, F. \& THØGERSEN, J. (1995): "Understanding of consumer behaviour as a prerequisite for environmental protection", Journal of consumer policy, 18(4), 345-385.

PARLAMENTO EUROPEO (2006): Report on Corporate Social Responsibility: a New Partnership (2006/2133(INI)),

http://www.europarl.europa.eu/meetdocs/2004_2009/documents/a6/p6_a(2006)0471_/p6_a(200 6)0471_en.pdf.

PETTY, R.E. \& CACIOPPO, J.T. (1984): "The effects of involvement on responses to argument quantity and quality: Central and peripheral routes to persuasion", Journal of Personality and Social Psychology, 46, 69-81.

PETTY, R.E., CACIOPPO, J.T. \& GOLDMAN, R. (1981): "Personal involvement as a determinant of argument-based persuasion", Journal of Personality and Social Psychology, 41, 847-855.

SHAW, D.S., HOGG, G., WILSON, E., SHIU, E. \& HASSAN, L. (2006): "Fashion victim: the impact of fair trade concerns on clothing choice", Journal of Strategic Marketing, 14(4), 427-440. 
STIGLER, G.J. (1961): "The Economics of Information", Journal of Political Economy, 69, $213-225$.

STO, E. \& STRANDBAKKEN, P. (2005): "Ecolabels and Consumers". In: Rubik, R. \& Frankl, P. (Ed.), The future of Ecolabelling. Making Environmental Product Information Systems Effective, Greenleaf Publishing, Londres, 92-119.

THØGERSEN, J., HAUGAARD, P. \& OLESEN, A. (2010): "Consumer responses to ecolabels", European Journal of Marketing, 44, 11/12, 1.787-1.810.

UUSITALO, O. \& OKSANEN, R. (2004): "Ethical Consumerism: A View from Finland", International Journal of Consumer Studies, 28(3), 214-221.

VALOR, C. (2007): "Influence of the Information about Labour Abuses on Consumer Choice of Clothes: A Grounded Theory Approach", Journal of Marketing Management, 23(7/8), 675-695.

VALOR, C. \& CALVO, G. (2009): "Compra responsable en España. Comunicación de atributos sociales y ecológicos", Información Comercial Española, Boletín Económico, 2.971, 33-50.

VAN AMSTEL, DRIESSEN, P. \& GLASBERGEN, P. (2008): "Eco-labeling and information asymmetry: a comparison of five eco-labels in the Netherlands", Journal of Cleaner Production, 16(3), 263276.

WEBB, D.J., MOHR, L.A. \& HARRIS, K.A. (2008): "A re-examination of socially responsible consumption and its measurement", Journal of Business Research, 61(2), 91-98.

ZUCKERMAN, A. \& CHAIKEN, S. (1998): "A heuristic-systematic processing analysis of the effectiveness of product warning labels", Psychology and Marketing, 15, 621-642. 